\title{
UVMag: a UV and optical spectropolarimeter for stellar physics
}

\section{Coralie Neiner ${ }^{1}$, Pascal Petit ${ }^{2}$, Laurent Parès ${ }^{2}$ and the UVMag consortium}

\author{
${ }^{1}$ LESIA, UMR 8109 du CNRS, Observatoire de Paris, UPMC, Univ. Paris Diderot, 5 place \\ Jules Janssen, 92195 Meudon Cedex, France \\ email: coralie.neiner@obspm.fr \\ ${ }^{2}$ IRAP, CNRS, UPS-OMP, 14 Avenue Edouard Belin, 31400 Toulouse, France
}

\begin{abstract}
UVMag is a space project currently under R\&D study. It consists in a mediumsize telescope equipped with a spectropolarimeter to observe in the UV and optical wavelength domains simultaneously. Its first goal is to obtain time series of selected magnetic stars over their rotation period, to study them from their surface to their environment, in particular their wind and magnetospheres. As the star rotates it will be possible to reconstruct 3D maps of the star and its surroundings. The second goal of UVMag is to obtain two observations of a large sample of stars to construct a new database of UV and optical spectropolarimetric measurements.
\end{abstract}

Keywords. instrumentation: polarimeters, instrumentation: spectrographs, ultraviolet: stars, stars: magnetic fields, stars: winds, outflows, stars: activity, stars: chromospheres

\section{Science drivers}

Research on stellar magnetism has been progressing very fast from the ground in the last decade, but we are missing information on stellar wind and magnetospheres because there is no UV spectrograph currently available for long fractions of time. To reconstruct the magnetospheres, we need to obtain simultaneous UV and optical spectropolarimetry, continuously over several stellar rotation periods. Of course, the UV domain requires a space mission. Therefore the UVMag consortium proposes to build a dedicated Msize space mission with a telescope of $1.3 \mathrm{~m}$ and UV and optical spectropolarimetric capabilities (see http://lesia.obspm.fr/UVMag).

The UV and visible spectropolarimeter will provide a very powerful and unique tool to study most aspects of stellar physics in general and in particular for stellar formation, structure and evolution as well as for stellar environment. For example we plan to study how fossil magnetic fields confines the wind of massive stars and influences wind clumping, how magnetic interactions impact binary stars, how a solar dynamo impacts its planets and how it evolves, how magnetic field, wind and mass-loss influence the late stages of stellar evolution, in which conditions a magnetic dynamo develops, how the angular momentum of stars evolves, how small-scale and large-scale stellar dynamos work and how their cycles influence their environment, what explains the diversity of magnetic properties in $\mathrm{M}$ dwarfs, what causes the segregation of tepid stars in two categories: those with sub-Gauss magnetic fields and those with fields above a few hundreds Gauss, what are the timescales over which magnetospheric accretion stops in PMS stars, etc. These questions will be answered by observing all types of stars: massive stars, giants and supergiants, chemically peculiar stars, pre-main sequence stars, cool stars, solar twins, M dwarfs, AGB and post-AGB stars, binaries, etc. Additional possible science includes the study of the ISM, white dwarfs, novae, exoplanets, atomic physics,... 


\section{UV and optical spectropolarimeter}

The spectropolarimeter should ideally cover the full wavelength range from 90 to $1000 \mathrm{~nm}$ and at least the most important lines in the domains $117-320 \mathrm{~nm}$ and 390$870 \mathrm{~nm}$. Polarisation should be measured at least in Stokes V (circular polarisation) in spectral lines, but the aim is to measure all Stokes QUV parameters (circular and linear polarisation) in the lines and continuum. A high spectral resolution is required, at least 25000 in the UV domain and at least 35000 in the optical, with a goal of 80000 to 100000 . The signal-to-noise should be above 100 .

Spectroscopy with these specifications in the UV and optical domains is relatively easy to achieve with today's technology and detectors. However, (1) high-resolution spectropolarimetry of stars has never been obtained from space; (2) optical spectropolarimeters available on the ground are large; and (3) it is very important to keep the instrumental polarisation at a low level. Therefore we have started a R\&D program to study a space UV+optical spectropolarimeter. Our study is based on existing ground-based spectropolarimeters, such as ESPaDOnS or Narval, and new spectropolarimetric techniques proposed in the literature (e.g. Sparks et al. 2012).

\section{Observing program}

UVMag will observe all types of stars in the magnitude range at least $V=3-10$. The observing program includes two parts: (1) 50 stars will be observed over 2 full rotational cycles with high cadence in order to study them in great details and reconstruct $3 \mathrm{D}$ maps of their surface and environment. In addition, the solar-like stars among those will be re-observed every year to study their activity cycle; and (2) two spectropolarimetric measurements of $\sim 4000$ stars will be obtained to provide information on their magnetic field, wind and environment. This will form a statistical survey and provide input for stellar modelling. The acquisition of the data for these two programs will take 4 years.

\section{Conclusions}

The UVMag consortium has set the basic requirements for a M-size $(1.3 \mathrm{~m})$ space mission to study the magnetospheres and winds of all types of stars. This is the next step to progress on the characterisation and modelling of stellar environments, as well as on important questions regarding stellar formation, structure and evolution. Simultaneous UV and optical spectropolarimetry over long periods of time is indeed the only way to comprehend the full interaction between various physical processes such as the stellar magnetic field and stellar wind. A R\&D study is ongoing for the instrument. The M-size mission will be proposed at ESA. A L-size mission (4-8 meter telescope) is also considered with the UVMag UV and optical spectropolarimeter as part of a series of instrument, e.g. on EUVO (Gómez de Castro et al., 2013).

\section{Acknowledgements}

The UVMag R\&D program is funded by the French space agency CNES.

\section{References}

Gómez de Castro, A. I., Appourchaux, T., Barstow, M., Barthelemy, M., Baudin, F., et al. 2013, $\operatorname{arXiv,~} 1306.3358$

Sparks, W., Germer, T. A., MacKenty, J. W., \& Snik, F. 2012, Applied Optics, 51, 5495 